\title{
Electron Paramagnetic Resonance Studies on Nitrogenase
}

\section{The Properties of Molybdoferredoxin and Azoferredoxin}

\author{
GRAHAM PAIALR, JAGIR S. MULTANI, WALTER C. CRETNEY \\ WALTER G. ZUMFT ${ }^{1}$ AND LEONARD E. MORTENSON
}

\begin{abstract}
Departmenl of Biological Chemislry and Biophysics Research Division, Instilute of Science and Technology, Iniversily of Michigan, Ann Arbor, Michigan 48105; and Department of Biological Sciences, Purdue Lniversity, Lafayatle, Indiana 47907
\end{abstract}

Received July 11, 1972

\begin{abstract}
Both molybdoferredoxin and azoferredoxin exhibit EPR signals. Reduced azoferredoxin has a $g=1.94$ type signal characteristic of nonheme iron proteins. Reduced molybdoferredoxin has EPR signals with $g$-values of $2.01,3.78$ and 4.27 . This resonance appears to be due to an antiferromagnetically coupled system of iron atoms with an effective spin of $3 / 2$ which has been subjected to both axial and rhombic distortions of the crystal field.

Oxidation of azoferredoxin with ferricyanide results in the disappearance of the $g$ $=1.94$ type signal and replacement by an isotropic line at $g \sim 2.0$. The EPR signals observed on oxidation of molybdoferredoxin depend on the extent of oxidation. The intensity of EPR lines at $g=3.78$ and 4.27 decreases on oxidation and the signal is finally replaced by a single line at $g=4.0$. The changes at high field on oxidation are more complex and three types of signals are observed. At a ferricyanide:protein ratio of $5: 1$, an isotropic line (I), superimposed on the $g \sim 2.01$ signal is observed. With a ratio of $20: 1$ the intensity of $I$ decreases to a negligible amount, but now three new signals at $g$ values of $1.76,1.90$ and 1.98 appear; they are part of rhombic trio (Species R). Excess ferricyanide reduces the intensity of R species, but produces an axial F.PR spectrum (A species) with $g_{\perp}=2.00$ and $g n=2.09$.
\end{abstract}

The fundamental importance of biological dinitrogen fixation has led to an intensive research effort in a number of laboratories. Studies have been focused mainly on the nitrogenase complexes from Azotobacter vinelandii, Clostridium pasteurianum and soybean nodules. Nitrogenase has been found to consist of two distinct protein components, azoferredoxin ( $\mathrm{F}_{\mathrm{e}}$ protein) and molybdoferredoxin (Mo-Fe protein), and these proteins have been purified from both $C$. pasteurianum and $A$. vinelandii. Azoforredoxin from C. pasteurianum is a dimeric nonheme iron-sulfur protein of molecular woight 55,000 containing four iron atoms and an equivalent amount of labile sulfide (1).

1 Research Associate sponsored by the Deutsche Forschungsgemeinschaft.
Molybdoferredoxin consists of two types of subunits with molecular weight of 59,500 and 50,700 , respectively $(2,3)$. In solution the protein exists as a tetramer with a molecular weight of 220,000 (3). The protein components from A. vinelandii (4) and Klebsiella pneumoniae (5) are quite similar in their physiochemical characteristies to those purified from C. pasteurianum.

Although some basic details of the reductive process have becn established, there is no understanding of either the role of the metal ions or the nature of interaction of various substrates and ATP with these proteins. This communication is the first in a sories of papors describing experiments designed to gain insight into the nature of ATP and substrate interaction with the metals 
present in molybdoferredoxin and azoferredoxin and reports on some of the $\mathrm{EPR}^{2}$ characteristics of the isolated proteins.

\section{MATERIALS AND METHOISS}

Molybdoferredoxin and azoferredoxin were purified by the procedure of Mortenson (6) modified to increase the resolution of the final DEAEcellulose chromatography (7). The purity of the proteins was checked by the SDS dise electrophoresis procedure of Nakos and Mortenson $(1,2)$ and the activity was measured using the acetylene reduction assay (7); molybdoferredoxin and azoferredoxin had acetylene reducing activities of 2300 and $2200 \mathrm{nmoles} / \mathrm{min} / \mathrm{mg}$ protein, respectively. Molybdoferredoxin contains 2 molybdenum and $22-24$ iron atoms $/ 220,000 \mathrm{~g}$ protein; azoferredoxin contains 4 iron atoms $/ 55,000 \mathrm{~g}$ protein (3). Protein concentration was estimated with the biuret reagent with bovine serum albumin (Sigma) as the standard.

EPR spectra were recorded on a Varian V4500 spectrometer modified to allow a greater dynamic range for microwave power (8). Samples were prepared in $4 \mathrm{~mm}$ o.d. quartz capillaries closed with serum caps. Prior to use the tubes were purged with $\mathrm{N}_{2}, \mathrm{H}_{2}$ or Ar which had been freed of oxygen by passing over hot copper turnings or BASF catalyst. All solutions were stored under the $\mathrm{O}_{2}$-free gas and were transferred to the EPR tubes using gastight Hamilton syringes. Mixing was accomplished by inverting and rotating the tubes numerous times.

EPR spectra were recorded at about $20^{\circ} \mathrm{K}$ as indicated the temperature being monitored by a carbon resistor mounted below the sample tube. The temperature differential between this resistor and a second one located at the sample position was ehecked frequently; the latter was consistently $2^{\circ} \mathrm{K}$ warmer than the monitoring resistor and this correction has been applied to the recorded temperature. $g$-Values were determined by recording spectra of the sample and of standard field markers. There were strong pitch for the $g=$ 2.0 region and $\mathrm{Cr}^{3+}$ doped into cobalt ethylenediaminetetra-acetate for the low field region (9). The latter material was a gift from Dr. R. Aasa to Dr. J. A. Fee. The magnetic field was independently calibrated with an Alpha model 3193 digital gaussmeter.

\section{RESULTS}

In Fig. 1 A, B we show the EPR spectra of reduced azoferredoxin and molybdoferredoxin; the signals are extremely temperature dependent and broaden rapidly above $30^{\circ} \mathrm{K}$

\footnotetext{
${ }^{2}$ Abbreviations: EPR, electron paramagnetic resonance.
}

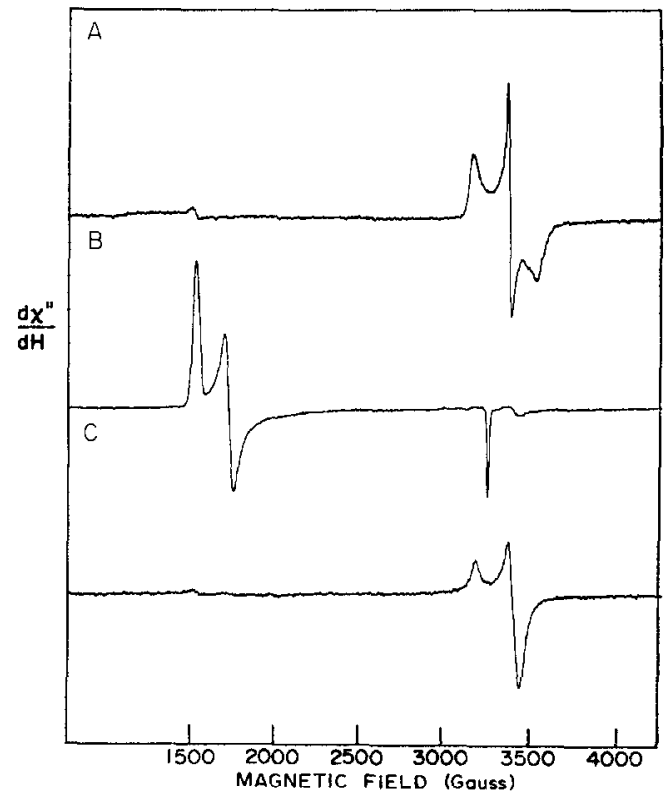

FIG. 1. EPR spectra of: (A) azoferredoxin $(20 \mathrm{mg} / \mathrm{ml}$, gain $=50)$, (B) molybdoferredoxin $(62 \mathrm{mg} / \mathrm{ml}$, gain $=25)$ and $(\mathrm{C})$ "impurity protein" $(23 \mathrm{mg} / \mathrm{ml}$, gain $=50)$ at $23^{\circ} \mathrm{K}$. Sweep rate, 500 $\mathrm{G} / \mathrm{min}$; time constant, $0.3 \mathrm{sec}$; modulation amplitude, $3.5 \mathrm{G}$.

so that by $60^{\circ} \mathrm{K}$ only a weak, poorly resolved spectrum is observed.

The spectrum of azoferredoxin (Fig. 1A) appears to be the simpler of the two proteins. Apart from a very small peak at $g=4.3$, presumably because of some minority species of iron, the spectrum consists of an approximately rhombic resonance of the $g=1.94$ type. The spectrum is clearly more complex than the simple rhombic spectrom observed with spinach ferredoxin (10) as judged by the extreme narrowness of the center line compared to the high and low field extrema and this suggests that there might be several species contributing to the overall line shape. The nominal $g$-values are $2.06_{5}, 1.94_{2}$ and $1.87_{1}$; these are close to the values reported for the protein from $K$. pneumoniae, viz., $2.05,1.93$ and 1.87 (5). The integrated intensity of this spectrum accounts for 0.2 electrons/mole of protein $\left(20^{\circ} \mathrm{K}, \mathrm{Cu}\right.$-EDTA standard, assuming $S=1 / 2$ ). If precautions are not taken to ensure an adequate concentration of dithionite in the preparative buffers, the intensity of the resonance is significantly reduced but can be restored by the subsequent addition of dithionite. 
The EPR spectrum of isolated molybdoferredoxin with an activity of 2300 units (Fig. 1B) exhibits a very narrow notch at $y=2.01$ and two very intense lines at 4.27 and 3.78. These three EPR resonances are most intense in the presence of excess dithionite and disappear in parallel following addition of oxidizing agents. As discussed below we believe these three lines are the three component $g$-values of a rhombic trio.

The quantitation of the intensity of the EPR spectrum of molybdoferredoxin is frustrated by: (i) the absence of an appropriate standard, (ii) the problem of making an accurate correction for the transition probability and (iii) the uncertainty of the spin state of the specios. By assuming that the resonance arises from the $\pm 1 / 2$ Kramer's doublet of an $S=3 / 2$ system (see Discussion) and estimating the correction to the transition probabilities by the method of Aasa and Vanngard (11) we find 0.5 moles paramagnet/mole of protein at $20^{\circ} \mathrm{K}$ using $\mathrm{Cu}^{\mathrm{II}}$-EDTA as a standard. It is not obvious that the correction for the transition probability is valid for this systcm and we are currently investigating this point using computer simulation of the spectra.

For reasons which are discussed bclow we originally believed that this resonance was because of $\mathrm{Mo}^{3+}$. For that reason we grew $C$. pasteurianum on a medium in which the molybdenum was supplied as the ${ }^{95} \mathrm{Mo}$ ( $\mathrm{I}=5 / 2$ ) isotope and isolated the molybdoferredoxin in the usual manner. The extent of incorporation of the isotope was checked by chemical degradation of the protein and subsequent mass spectrometric analysis; the isotopically enriched molybdoferredoxin was found to contain $76 \%$ of the ${ }^{95} \mathrm{Mo}$ isotope.

The EPR spectra of the enriched and isotopically normal molybdoferredoxins were recorded at $13^{\circ} \mathrm{K}$. There were no differences between the spectra at any of the three $g$ values. The full line width at half height of the $g=2.01$ component is about $16 \mathrm{G}$ and it should have been readily possible to detect a line broadening of $5 \mathrm{G}$ equivalent to a hyperfine interaction of ca. $1 \mathrm{G}$. Neither broadening nor additional satellite lines could be detected in the enriched protein.

In samples of molybdoferredoxin of lower activity an additional resonance of the $g=$
1.94 type is observed (cf. 4); this material appears to be a catalytically inactive molybdoferredoxin which can be separated from native molybdoferredoxin by DEAE chromatography (7). This second species is a molybdenum-free iron-sulfur protein and has a relatively simple EPR spcctrum (Fig. 1C) with nominal $g$-values $g_{\perp}=1.92_{5}, g_{\|}=$ $2.05_{7}$ though the line shape is not accurately axial. The integrated intensity of this resonance accounts for 0.13 moles of unpaired electron $/ 10^{5} \mathrm{~g}$ of protein (assuming $S=1 / 2$ ). The protein is very sensitive towards oxidizing agents and addition of only a small amount of ferricyanide causes the complete disappearance of the signal. The EPR intensity of this protein saturates rather readily as the temperature is lowered below $20^{\circ} \mathrm{K}$; this is in contrast to the $4.27,3.78$, 2.01 species which saturates with difficulty even at the lower temperature. As a result data taken at low temperature and high microwave power show little if any of this impurity (Fig. 2).

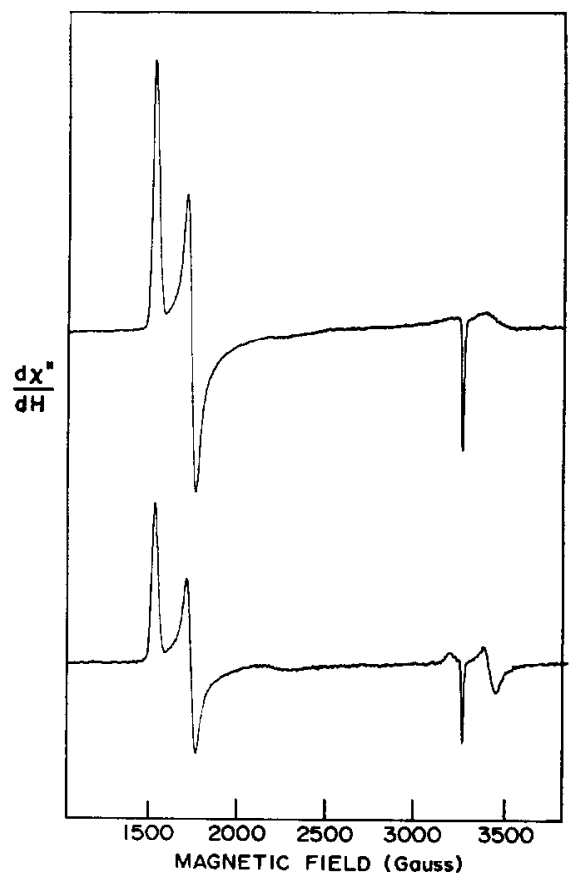

FIG. 2. Effect of temperature on the relative intensities of molybdoferredoxin and the impurity protein: (top) $7^{\circ} \mathrm{K}$, gain $=25$; (bottom) $222^{\circ} \mathrm{K}$, gain $=50$; sweep rate, $500 \mathrm{G} / \mathrm{min}$; time constant, $0.3 \mathrm{sec}$; modulation amplitude, $3.5 \mathrm{G}$, frequency, $9.22 \mathrm{GHz}$. 


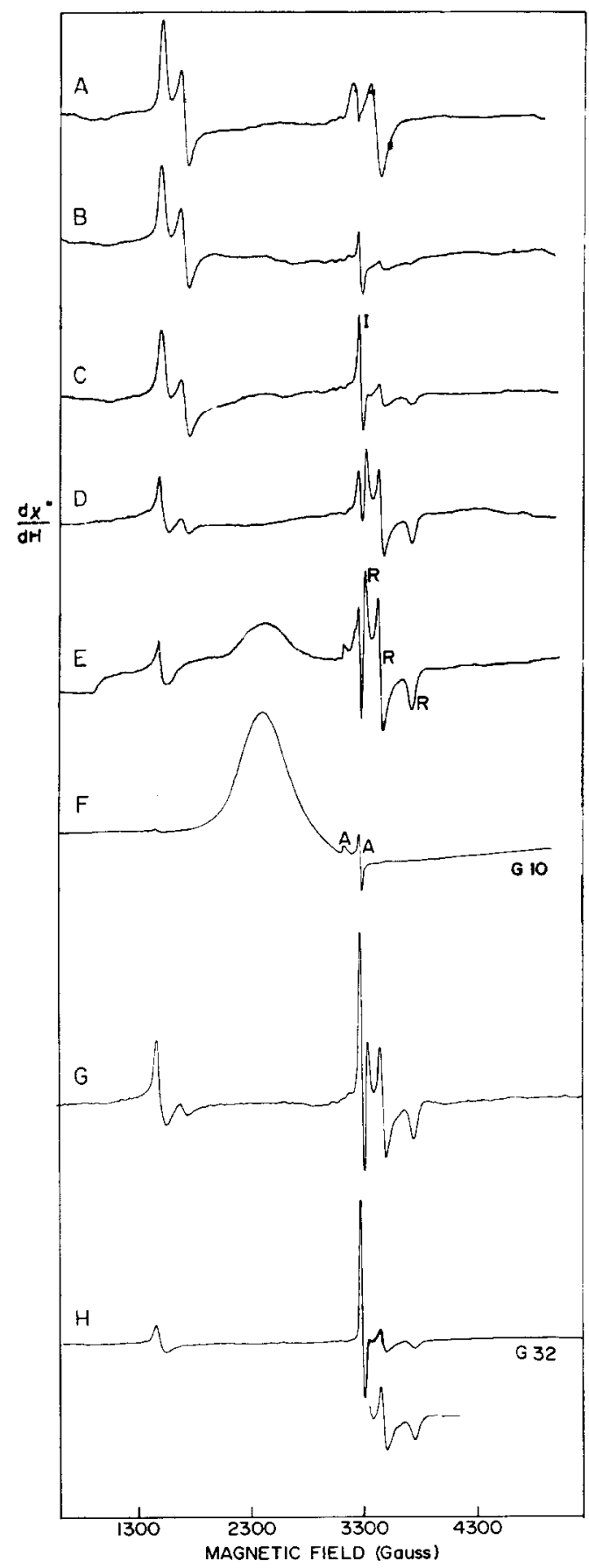

Fig. 3, Effect of oxidants on molybdoferredoxin $(60 \mathrm{mg} / \mathrm{ml})$ : (A) Reduced molybdoferredoxin: note the presence of the substantial amount of impurity in the $g=2$ region and the almost complete obliteration of the $g=2.0$ notch by the fast sweep rate and relatively long time

\section{Effect of Oxidizing Agents}

We have studied the effects of two oxidants; viz, oxygen and ferricyanide, on these proteins. Because experiments with ferricyanide are easier to control most of our results are with this reagent.

The simplest results are obtained with reduced azoferredoxin. Addition of potassium ferricyanide, tenfold over protein, completely eliminates the $g=1.94$ nonheme iron signal and produces a narrow asymmetric signal in the $g=2.0$ region which is reduced in amplitude by further addition of the oxidizing agent while the small $g=4.3$ signal increases slightly in amplitude. Similar changes are observed when the protein is exposed to air for $5 \mathrm{~min}$.

The changes that occur when ferricyanide is added to molybdoferredoxin are more complicated. Low concentrations of the oxidant $\left(\mathrm{Fe}(\mathrm{CN})^{3-}{ }_{6} /\right.$ protein $=$ about $5 / 1$ molar ratio $)$ produce a small, almost isotropic, absorption in $g=2$ region (Fig. 3B) which presumably consists of the high field extremum of the low field signal superimposed with a new narrow resonance I (isotropic). The intensity of the low field doublet decreases by some $40 \%$. Doubling the ferricyanide concentration decreases the intensity of the doublet a little more while that of species I increases by a factor of 2 and additional resonances become observable at $g \sim 1.76$ and 1.90 (Fig. 3C). Further additions of ferricyanide produce an increase in the intensity of these latter resonances while species I decreases to an insignificant amount and this allows observations of a third resonance at $g \sim 1.98$; this

constant. (B) After addition of potassium ferricyanide, five-fold over protein. Note the species I. (C) Ferricyanide now tenfold over protein. (D) Ferricyanide now 20 -fold over protein. (E) Ferricyanide now 30 -fold over protein. Note species $R$ and the presence of the broad resonance at ca. $2400 \mathrm{G}$ typical of oxidized potassium ferricyanide. (F) Large excess of potassium ferricyanide. (G) Molybdoferredoxin after $5 \mathrm{~min}$ incubation with air. (H) Additional $25 \mathrm{~min}$ incubation with air. Sweep rate, $100 \mathrm{G} / \mathrm{min}$; time constant, 1 sec; modulation amplitude, $3.5 \mathrm{G}$ : frequency, $9.2 \mathrm{GHz}$; gain $=100$ (unless indicated otherwise); temp, $23^{\circ} \mathrm{K}$. 
appears to be the low ficld components of a rhombic trio with $g$-values of $1.76,1.90$ and 1.98 (we call this species R) (Fig. 3D and E).

Additional inerements of ferricyanide lead to) a reduction in the intensity of $R$ and the appearance of yet a third species exhibiting an axial EPR spectrum with $y_{\perp}=2.00, y_{2}=$ 2.09 ; we call this species $\mathrm{A}$. With excess forricyanide, R (Fig. 3F) has completely disappeared while $A$ has its maximum intensity; it should be emphasized that the protein is consuming ferricyanide throughout most of this experiment for the EPR of ferricyanide is only observed with the appearance of A. Addition of excess dithionite at this point restores about $50 \%$ of the low field and nonheme iron signals.

When molybdoferredoxin is exposed to air for 5 min (Fig. 3G) the spectrum that is observed is similar to that produced by intermediate amounts of ferricyanide. The low field doublet is replaced by a singlet ( $g=$ 4.0 ), and the $g=2$ region consists of both $I$ and $R$ species with comparable amplitude. lurther exposure to air (Fig. 3H) increases the intensity of species I markedly whereas the intensity of $R$ decreases by about $30 \%$ and the low field region loses the remains of the doublet and now exhibits only the single resonance.

Weakly oxidizing redox dyes such as methylene blue or phenazine methosulfate do not produce these complex changes. With molybdoferredoxin both methylene blue and phenazine methosulfate climinate the signals present in the reduced enzyme. With azoferredoxin phenazine methosulfate serves as oxidant, methylene blue was not examined becuuse its free radical species is large and overlaps the $g=1.94$ region.

\section{DISCUSSION}

Because of their sensitivity to oxygen the proteins of the nitrogenase complex are isolated in the presence of $1 \mathrm{mu}$ dithionite (7). Under these conditions both molybdoferredoxin and azoferredoxin exhibit EPR spectra which are not obviously similar to the spectra observed with any other protein.

Azoferredoxin exhibits a resonance which is of the general type called $\%=1.94$ and which is diagnostic of reduced iron-sulfur protein such as the plant and bacterial ferredoxin $(10,12)$. To the extent that the envelope of the resonance does not conform to any of the simple axial or rhombic prototypes it is possible that the resonance is heterogeneous, that is there are at least two paramagnetic centers contributing to the EPR intensity as has been shown previously for bacterial ferredoxin (13). In this latter case there are two active centers each containing four iron atoms, four sulfide ions and four cysteine residues (14); azoforredoxin contains only four iron atoms and thus if this protein contains two active centers cach with iron exhibiting EPR in the reduced state, then each active conter must contain two iron atoms, that is azoferredoxin may be a dimer of a "spinach-ferredoxin type" active center. This possibility however is inconsistent with the optical propertics of azoferredoxin; the protein does not have the reddishbrown color typical of the $2 \mathrm{Fc} \sim \mathrm{S}^{*}$ proteins rather, it exhibits a greenish color which is closer to that observed with the bacterial ferredoxins. Thus the anomalous line shape of azoferredoxin may be the consequences of a yet-to-be explained feature of the paramagnetic properties of a tetrahedral cluster of iron atoms. The integrated intensity of the EPR is surprisingly low, we had expected to find 1 electron/mole protein. If the protein were impure then two possibilities exist: (a) the EPR is from the impurity or (b) the EPR is from azoferredoxin but most of the protein is contaminant. However the preparations we have used show a single sharp band on SDS gel which makes it unlikely that any significant amount of a second protein is present. Furthermore in a subsequent paper (15) we will report on the response of this EPR signal to $\mathrm{MgATP}$ and on rapid-freezing studies during turnover which clearly establish that this EPR species is a physiologically important component of the nitrogenase system. At the present time we are unable to provide a satisfactory explanation for the low EPR integration.

The EPR spectrum of isolated molybdoforredoxin of very high activity is of simple shape though the EPR parameters are quite unique. A similar spectrum has been observed in proteins isolated from $A$. vinelandii 
$(4,16)$ and $K$. pneumoniae $(5)$ and in whole cells of A. vinelandii, C. pasteurianum, Bacillus polymyxa and $K$. pneumoniae (16).

The three resonances at $g=4.27,3.78$ and 2.01 have the appearance of a rhombic trio and can be explained as being due to a $S=$ $3 / 2$ system. A system containing three unpaired electrons will be sensitive to zero field splitting if subjected to noncubic crystal fields. If this crystal field has strong axial asymmetry $(D \gg g B H$, where $D$ is the axial crystal field splitting parameter), then one would predict an EPR spectrum with $g_{\perp}=$ 4.0 and $g_{\mathbb{1}}=2$. A secondary, rhombic, distortion $(0<E / D<1 / 3$, where $E$ is the rhombic field splitting parameter) will split the low field resonance into two components approximately symmetrically located about $g_{\perp}$. We thus propose that the lines at 4.27 , 3.78 and 2.01 are the $g_{x}, g_{y}$ and $g_{z}$ of a $S=3 / 2$ system subjected to both axial and rhombic zero field splittings.

One obvious candidate for the $S=3 / 2$ species is the molybdenum component of molybdoferredoxin which in the $3^{+}$valence has the $4 d^{3}$ electronic configuration; an EPR spectrum of the type observed has been previously reported for Mo(III) acetylacetonate (17). However, if this species is indeed molybdenum one would expect to see molybdenum hyperfine structure from the $\mathrm{I}=5 / 2$ isotopes which are present to about $25 \%$ in natural abundance. We have been unable to detect any such hyperfinc lines in the native protein or in a protein in which ${ }^{95} \mathrm{Mo}$ had been enriched to $76 \%$. We are not aware of any compound of molybdenum which exhibit a sufficiently narrow hyperfine structure to explain our results. On the contrary the molybdenum hyperfine interaction is substantial, typically $40-70 \mathrm{G}$, and we are thus forced to conclude that the $S=3 / 2$ species arises from the iron and not the molybdenum component of molybdoferredoxin.

Mononuclear iron is rarely found in the $S=3 / 2$ state and even then this can only occur with ferric iron. The rhombic resonance, however, is maximal in the presence of dithionite and thus the probability that it is mononuclear ferric iron is remote. The remaining alternative is that the resonance is from a polynuclear system of iron atoms which are exchange-coupled to yield a $S=3 / 2$ ground state, in much the same way that the ferric and ferrous ions in reduced spinach ferredoxin couple to yield a $S=1 / 2$ ground state $(18,19)$. Exchange coupling to give an $S=3 / 2$ ground state requires that the polynuclear center contain at least three iron atoms with the possible configurations $3 \mathrm{Fe}^{3+}$ or $2 \mathrm{Fe}^{2+}$ plus $1 \mathrm{Fe}^{3+}$. With more than three iron atoms the alternative configurations are many. Molybdoferredoxin contains about 12 iron atoms/ $110,000 \mathrm{MW}(3)$.

For reasons specified earlier we do not have an accurate integration on the molybdoferredoxin resonance. However the preliminary estimate recorded in the experimental section makes it clear that the resonance is not because of some minority species which could be postulated as a contaminant in the preparation. Again, the absence of any impurity as judged by SDS gel electrophoresis and the behavior of molybdoferredoxin during rapid-freezing turnover experiments (15) makes it slear that this is a physiologically important component of nitrogenase.

Evans et al. (20) have recently reported some EPR spectra of the molybdoferredoxin from Chloropseudomonas ethylicum, a green photosynthetic $\mathrm{N}_{2}$-fixing organism. Their data, which was confined to the $g=2$ region, is different from that reported here and by others $(4,5,16)$. The spectra show a radical and some iron-sulfur resonances with no evidence for the $g=2.01$ notch that is clcarly charactcristic of most types of molybdoferredoxin. One is forced to conclude that either the preparation of Evans el al. is grossly impure or that the molybdoferredoxin present in Chloropseudomonas is fundamentally different to that from Clostridium. In support of the latter possibility Evans et al. demonstrated that the EPR of their protein was modified by both ATP and by ATP plus $\mathrm{CN}^{-}$while the EPR spectrum of clostridial molybdoferredoxin is not changed by ATP (15).

It is worth noting that the 1.94 resonance seen in less active fractions of molybdoferredoxin were not detected in whole cells at $4.2^{\circ} \mathrm{K}$ and were only just discernible at $13^{\circ} \mathrm{K}$ (16). This is a reflection of the relative saturation characteristics of the molybdoferredoxin and 1.94 resonances which are such that at very low temperatures the EPR of 
the former protein will be emphasized considerably over the much more readily saturated 1.94 resonance (cf. Fig. 2); this behavior should be noted when using EPR to examine the purity of molybdoferredoxin.

Both azoferredoxin and molybdoferredoxin are exceedingly sensitive to oxygen, a fact which delayed isolation of these proteins until well-controlled procedures for anaerobic purification were developed (6). The reaction of these proteins with oxidants is extremely complex. Redox dyes of relatively low potential such as methylene blue are either inert or oxidize the protein to what is assumed to be the physiological oxidized state. However oxidants of high potential, viz, ferricyanide or oxygen can initiate a complex series of reactions. This is best documented with molybdoferredoxin in which three discrete oxidized species are discernible. On the basis of their EPR lineshapes these have been designated I (isotropic), $\mathrm{R}$ (rhombic) and (Aaxial), the sequence of appearance being $I, R, A$ as the ferricyanide concentration is increased; spccics $A$ appears to be stablc to an cxcess of ferricyanide.

The reaction of molybdoferredoxin with air yields a mixture of I and R; presumably there is a kinetic barrier to the subsequent formation of $\mathrm{A}$. In view of our ignorance of the structure of the active center of molybdoferredoxin it seems premature to speculate on the nature of the species that we observe. Radicals of nitrogen exhibit essentially isotropic resonances at $g=2.00$ which exhibit nitrogen hyperfine structure (21) and are thus unlikely candidates. A possible participation of the "impurity" protein cannot be ruled out though we have not detected any correlation between the amount of this contaminant and the intensity of the $A, I$ and $R$ resonances. Further, the addition of ferricyanide to the "impurity" protein eliminates its $g=1.94$ resonance, but no new signals are observed. It should, perhaps, be stressed that when the intrinsic signals of molybdoferredoxin are abolished during turnover no new resonances of the type $\mathrm{A}, \mathrm{I}$ or $\mathrm{R}$ are seen in the $g=2.0$ region (15). However, it should be noted that with both the $2 \mathrm{Fe}$ and $8 \mathrm{Fe}$ ferredoxins reaction with ferricyanide and oxygen has been shown to oxidize the labile sulfide-cysteine system to sulfur-zero and this type of reaction has been postulated to be responsible for the oxygensensitivity of the iron-sulfur proteins (22).

\section{ACKNOWLEDGMENTS}

Excellent technical assistance of Claudette Polis, Richard Bare and Dottie Sheets in the purification of proteins is highly appreciated, and we are indebted to Drs. II. Brintzinger and W. II. Orme-Johnson for some valuable discussion. This work was supported by Research Grants GM 12176 (G. P.) and AI 04865 (L. F. M.) from the National Institutes of Health.

\section{REFERENCES}

1. Nakos, G., and Mortenson, L. E., (1971) Biochemistry 10, 455.

2. Nakos, G., and Mortenson, L. E., (1971) Biochim. Biophys. Acta 229, 431.

3. Hu.sng, T. C., Zumft, W. G., ANd Mortenson, L. E. unpublished data.

4. HARdy, R. W. F., BuRns, R. C., AND PARShalL, G. W. in Bio Inorganic Chemistry, Advances in Chemistry Series No. 100, (1971) Amer. Chem. Soc., Washington DC, p. 219.

5. EAdY, R. R., SмITH, B. E., СooK, K. A., AND Postgate, J. R. (1972) Biochem. J., 128, 655.

6. Montenson, L. E. (1972) in Methods in Enzymology (San Pietro, A., ed.), Vol. 23B, Academic Press, New York.

7. Zumet, W. G., and Mortenson, L. E. unpublished data.

8. Palmer, G. (1967) in Methods in Enzymology, (Estabrook, R. W., and Pullman, M. E., eds.), Vol. 10, p. 594, Academic Press, New York.

9. Alsa, R., Falk, K. E., and Reyes, S. A. (1966) Ark. Kemi 25, 309.

10. Palmer, G., and Sands, R. H. (1966) $J$. Biol. Chem. 241, 253.

11. Aasa, R., and Vanngard, T. (1964) Z. Naturforsch. 19, 1425.

12. Palmer, G., Sands, R. H., and Mortenson, L. E. (1966) Biochem. Biophys. Res. Commun. 23,357 .

13. Orme-Johnson, W. H., and Beiner't, H. (1969) Biochom. Biophys. Res. Commun. 36, 337.

14. Sieker, L. C., Adman, E., And Jensen, L. H. (1972) Nature (London) 235, 40.

15. Mortenson, L. E., Zumft, W. G., and Palmer, G. (1972) Biochim. Biophys. Acta, in press.

16. Divis, L. C., Shah, V. K., Brile, W. J., AND Orme-Johnson, W. H. (1972) Biochim. Biophys. Acta 256, 512.

17. J.rrete, H. S. (1957) J. Chem. Phys. 27, 1298 
18. Dunham, W. R., Palmer, G., Sands, R. H., and Bearden, A. (1971) Biochim. Biophys. Acta 253, 373.

19. Gibson, J. F., Hall, D. O., Thornley, J. H. M., ANd Whatley, F. R. (1966) Proc. Nat. Acad. Sci. USA 56, 987.

20. Evans, M. C. W., Telfer, A., Cammack, R.
ANd Smith, R. V. (1971) FEBS (Fed. Eur. Biochem. Soc.) Lett. 15, 317.

21. Atkins, P., And Symons, M. C. R. (1967) in The Structure of Inorganic Radicals, Elsevier, London.

22. Petering, D., Fee, J. A., and Palmer, G. (1971) J. Biol. Chem. 246, 643. 